\title{
Ipsilateral Prominent Thalamostriate Vein on Susceptibility- Weighted Imaging Predicts Poor Outcome after Intravenous Thrombolysis in Acute Ischemic Stroke
}

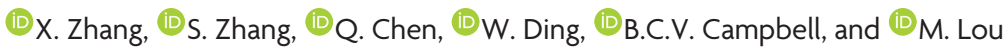

\begin{abstract}
BACKGROUND AND PURPOSE: The impact of deep cerebral veins on neurologic outcome after intravenous thrombolysis in patients with acute ischemic stroke is unclear. We investigated the relationship between the appearance of deep cerebral veins on susceptibilityweighted imaging and neurologic outcome in patients who underwent thrombolysis.
\end{abstract}

MATERIALS AND METHODS: We retrospectively analyzed 109 consecutive patients with acute ischemic stroke who had pretreatment SWI and received intravenous thrombolysis within 6 hours. We calculated the signal difference ratio (defined as the relative difference in signal intensity between the ipsilateral and contralateral veins) of the thalamostriate vein, septal vein, and internal cerebral vein on pretreatment SWI.

RESULTS: Only the signal difference ratio of the thalamostriate vein was significantly associated with poor outcome (3-month modified Rankin Scale score $>2, P=.008)$. The optimal threshold was relative hypointensity of the ipsilateral vein of $>4.8 \%$ (sensitivity of $53.7 \%$ and specificity of $80.9 \%$ ). We defined a signal difference ratio of the thalamostriate vein of $\geq 5 \%$ as an ipsilateral prominent thalamostriate vein. Patients with an ipsilateral prominent thalamostriate vein were more likely to have poor outcome $(\mathrm{OR}=3.66 ; 95 \% \mathrm{Cl}$, $1.25-10.68 ; P=.02$ ) and a lower rate of successful reperfusion (reperfusion rate of $\geq 70 \% ; \mathrm{OR}=0.35 ; 95 \% \mathrm{Cl}, 0.13-0.92 ; P=.03$ ), compared with those without an ipsilateral prominent thalamostriate vein. However, patients with an ipsilateral prominent thalamostriate vein were still less likely to experience poor outcome when reperfusion was achieved compared with when reperfusion did not occur $(80.0 \%$ versus $44.4 \%, P=.04)$.

CONCLUSIONS: A pretreatment ipsilateral prominent thalamostriate vein was associated with reduced reperfusion after thrombolysis and poor outcome. More intensive reperfusion approaches may be required for patients with an ipsilateral prominent thalamostriate vein.

ABBREVIATIONS: AIS = acute ischemic stroke; ICV = internal cerebral vein; IPTSV = ipsilateral prominent TSV; IVT = intravenous thrombolysis; SDR = signal difference ratio; $S V=$ septal vein; $T S V=$ thalamostriate vein

V enous changes in the affected hemisphere after acute ischemic stroke (AIS) may play a crucial role in determining clinical outcome. ${ }^{1-3}$ Susceptibility-weighted imaging is useful for

Received September 2, 2016; accepted after revision December 30.

From the Departments of Neurology (X.Z., S.Z., Q.C., M.L.) and Radiology (W.D.), the Second Affiliated Hospital of Zhejiang University, School of Medicine, Hang zhou, China; and Department of Neurology (B.C.V.C.), Royal Melbourne Hospital, Grattan St, Parkville, Victoria, Australia.

This work was supported by the Science Technology Department of Zhejiang Province (2013C13G2010032) and the National Natural Science Foundation of China (81471170\&81622017). The perfusion analysis software (MIStar) used in this study was provided to the site as part of their involvement in the International Stroke Perfusion Imaging Registry (INSPIRE; inspire.webmistar.com) study funded by the National Health and Medical Research Council of Australia.

Please address correspondence to Min Lou, MD, PhD, Department of Neurology, the Second Affiliated Hospital of Zhejiang University, School of Medicine, No. 88 Jiefang Rd, Hangzhou, China; e-mail: loumingxc@vip.sina.com

-- Indicates open access to non-subscribers at www.ajnr.org

http://dx.doi.org/10.3174/ajnr.A5135 evaluating cerebral veins in AIS because it is extremely sensitive to paramagnetic substances such as deoxyhemoglobin, thus reflecting the oxygen extraction fraction and the cerebral metabolic rate of oxygen of the hypoxic tissue. ${ }^{1-4}$ Shortly after vascular occlusion, the uncoupling between oxygen supply and demand in the hypoperfused region leads to an elevated oxygen extraction fraction and subsequent increased level of deoxyhemoglobin in the vessel, which contributes to prominent hypointensity of the draining veins on SWI. ${ }^{5-7}$

Asymmetric cortical veins, which can be visualized on SWI after AIS and are considered an indicator of salvageable ischemic penumbra, were found to be associated with 3-month outcome. ${ }^{4,8}$ However, it was difficult to differentiate cortical veins from leptomeningeal collaterals because there were no obvious morphologic differences between them. ${ }^{9}$ Additionally, the anatomic variability of cortical veins also contributes to reduced interrater agreement when assessing the cortical veins. In contrast to 


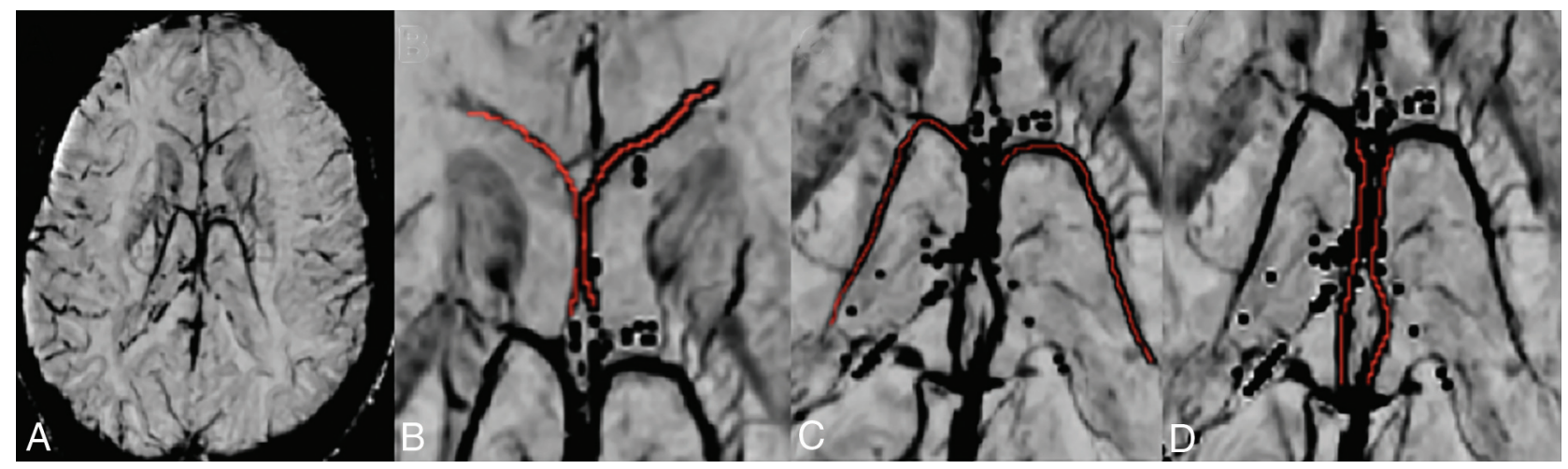

FIG 1. Veins selected for analysis on reconstructed susceptibility-weighted imaging. A, Reconstructed imaging in a minimum intensity projection of $20-\mathrm{mm}$ thickness. Septal vein $(B)$, thalamostriate vein $(C)$, and internal cerebral vein $(D)$. Red indicates the ROI.

cortical veins, leptomeningeal collaterals do not interfere with the assessment of deep cerebral veins, and anatomic variation in deep veins is less prevalent.

On the basis of SWI, it was demonstrated that prominent hypointense cortical veins and medullary veins predicted infarct growth and poor outcome after AIS. ${ }^{2}$ However, most of the included patients did not undergo revascularization therapy, and salvage of potentially penumbral regions was therefore unlikely. The imaging was also acquired up to 72 hours after AIS, which limits its applicability to hyperacute treatment decision-making. Furthermore, hypointense veins were only associated with prognosis in patients with large-vessel occlusion after AIS. Actually, slight perfusion abnormalities could even cause signal changes in the internal cerebral vein (ICV) and thalamostriate vein (TSV) on SWI. ${ }^{10}$

Therefore, we developed a quantitative method to assess signal asymmetry in the major deep cerebral veins, including the ICA, TSV, and septal vein (SV), and hypothesized that this could sensitively reflect cerebral metabolic changes in hypoxic brain tissue after AIS and may be associated with clinical outcome after intravenous thrombolysis (IVT).

\section{MATERIALS AND METHODS \\ Ethics Statement}

The protocol of MR imaging-guided intravenous thrombolysis has been approved by our local human ethics committee. All clinical investigations were conducted according to the principles expressed in the Declaration of Helsinki. Informed consent was obtained for all patients.

\section{Patient Selection}

We retrospectively reviewed our consecutive patients with AIS who received intravenous alteplase $(0.9 \mathrm{mg} / \mathrm{kg}$, up to a maximum of 90 $\mathrm{mg} ; 10 \%$ of the total dosage as a bolus and the rest infused for 1 hour) between June 2009 and July 2015. We then included patients under the following circumstances: 1) They received IVT within 6 hours of symptom onset; 2) underwent pretreatment multimodal MR imaging, including SWI, diffusion-weighted imaging, and perfusionweighted imaging; 3 ) had hypoperfusion (time-to-maximum of $>6$ seconds) in the supratentorial brain; and 4) underwent follow-up DWI or NCCT at 24 hours after IVT. Patients were excluded for the following reasons: 1) Their prestroke modified Rankin Scale score was $\geq 2 ; 2$ ) their MR imaging was degraded due to motion artifacts; or 3 ) there were bilateral perfusion lesions.

\section{Imaging Acquisition}

Multimodal MR imaging was performed on a 3T system (Signa Excite HD; GE Healthcare, Milwaukee, Wisconsin) equipped with an 8-channel phased array head coil. Foam pads were inserted into the space between the subject's head and the MR imaging head coil to minimize head motion. The MR imaging protocol included axial isotropic diffusion-weighted imaging ( $\mathrm{TR}=$ $4000 \mathrm{~ms}$; TE $=69.3 \mathrm{~ms}$; b-value $=1000 \mathrm{~s} / \mathrm{mm}^{2} ; \mathrm{FOV}=240 \mathrm{~mm}$; section thickness $=5 \mathrm{~mm}$; section gap $=1 \mathrm{~mm}$; acquisition matrix $=160 \times 160$; acquisition duration $=32$ seconds); TOF-MRA $\left(\mathrm{TR}=20 \mathrm{~ms} ; \mathrm{TE}=3.2 \mathrm{~ms}\right.$; flip angle $=15^{\circ}$; acquisition matrix $=$ $320 \times 224$; section thickness $=1.4 \mathrm{~mm} ; 3$ slabs, acquisition duration $=3$ minutes 46 seconds); SWI ( $\mathrm{TE}=4.5 \mathrm{~ms}$ [first echo]; interecho spacing $=4.5 \mathrm{~ms} ; \mathrm{TR}=58 \mathrm{~ms} ; \mathrm{FOV}=24 \times 24 \mathrm{~cm}^{2}$; matrix size $=256 \times 256$; flip angle $=20^{\circ}$; section thickness $=2.0$ $\mathrm{mm}$ with no gap between sections; acquisition duration $=3 \mathrm{~min}$ utes 27 seconds); and perfusion-weighted imaging (FOV $=240$ $\mathrm{mm} ; 50$ repetitions with $\mathrm{TR}=1500 \mathrm{~ms}$; $\mathrm{TE}=30 \mathrm{~ms}$; acquisition matrix $=128 \times 128$; section thickness $=5 \mathrm{~mm}$; section gap $=1$ $\mathrm{mm}$, gadolinium dose $=15 \mathrm{~mL}$; flow rate $=4-5 \mathrm{~mL} / \mathrm{s}$; acquisition duration $=1$ minute 15 seconds). The entire duration of the MR imaging protocol was 15 minutes.

\section{Imaging Analysis}

Assessment of Deep Veins on Pretreatment SWI. To assess venous structures, we reconstructed SWI in a minimum intensity projection of 20-mm thickness with OsiriX Imaging Software (http://www.osirix-viewer.com) (Fig $1 A$ ). As indicated in Fig $1 B-D$, the mean signal-intensity value of ICV, TSV, and SV was measured by drawing a line (1 pixel in width) along the center of each vein. After obtaining the contralateral mean signal-intensity value $\left(S_{\text {co-v }}\right)$ and ipsilateral mean signal-intensity value $\left(S_{\text {ip-v }}\right)$ of each vein, we calculated the signal difference ratio (SDR) by using the following equation:

$$
\operatorname{SDR}=\left(S_{\text {co-v }}-S_{\text {ip-v }}\right) / S_{\text {co-v }} \times 100 \% .
$$

Two stroke fellows (S.Z. and X.Z.) who were blinded to other imaging and clinical data independently assessed the SDR of SV, 


\begin{tabular}{|c|c|c|c|c|}
\hline & $\begin{array}{l}\text { Good Outcome (mRS } \leq 2) \\
\qquad(n=68)\end{array}$ & $\begin{array}{l}\text { Poor Outcome (mRS > 2) } \\
\qquad(n=41)\end{array}$ & Test Value & $P$ Value \\
\hline Age (yr) & $66.1 \pm 12.7$ & $72.0 \pm 12.4$ & $t=-2.36$ & $.02^{\mathrm{C}}$ \\
\hline Female (No.) (\%) & $21(30.9)$ & $19(46.3)$ & $\chi^{2}=2.63$ & .15 \\
\hline \multicolumn{5}{|l|}{ Risk factors } \\
\hline Hypertension (No.) (\%) & $46(67.6)$ & $29(70.1)$ & $\chi^{2}=0.11$ & .83 \\
\hline Diabetes mellitus (No.) (\%) & $12(17.6)$ & $13(31.7)$ & $\chi^{2}=2.86$ & .10 \\
\hline Atrial fibrillation (No.) (\%) & $21(30.9)$ & $21(51.2)$ & $\chi^{2}=4.47$ & $.04^{\mathrm{c}}$ \\
\hline Smoking (No.) (\%) & $30(44.1)$ & $13(31.7)$ & $\chi^{2}=1.65$ & .23 \\
\hline Previous stroke/TIA (No.) (\%) & $11(16.2)$ & $6(14.6)$ & $\chi^{2}=0.05$ & 1.00 \\
\hline Large-artery occlusion (No.) (\%) & $28(41.2)$ & $32(78.0)$ & $\chi^{2}=14.05$ & $<.001^{\mathrm{c}}$ \\
\hline Baseline SBP $(\mathrm{mm} \mathrm{Hg})$ & $155.4 \pm 20.5$ & $151.0 \pm 24.9$ & $t=0.64$ & .53 \\
\hline Baseline DBP (mm Hg) & $87.1 \pm 17.1$ & $83.2 \pm 14.8$ & $t=1.21$ & .23 \\
\hline ONT (min) & $251.2 \pm 74.8$ & $235.1 \pm 73.4$ & $t=-1.11$ & .27 \\
\hline Baseline glucose level (mmol/L) & $7.7 \pm 2.6$ & $8.1 \pm 2.7$ & $t=-0.77$ & .44 \\
\hline Baseline NIHSS score & $6.0(3.0-10.0)$ & $14.0(8.5-18.0)$ & $Z=-5.11$ & $<.001^{\mathrm{c}}$ \\
\hline Baseline diffusion lesion volume $(\mathrm{mL})$ & $1.0(0-14.2)$ & $8.4(2.9-51.2)$ & $Z=-3.12$ & $.002^{c}$ \\
\hline Baseline hypoperfusion volume (mL) & $22.7(3.0-74.6)$ & $81.8(31.9-166.6)$ & $Z=-4.16$ & $<.001^{\mathrm{C}}$ \\
\hline SDR of TSV (\%) & $1.2(-2.6-3.6)$ & $5.8(-1.1-8.9)$ & $Z=-2.61$ & $.009^{c}$ \\
\hline SDR of SV (\%) & $-0.3(-2.4-2.6)$ & $0.1(-3.0-3.7)$ & $Z=-0.45$ & .65 \\
\hline SDR of ICV (\%) & $-0.1(-4.1-3.5)$ & $0.9(-5.1-5.7)$ & $Z=-.71$ & .48 \\
\hline Proportion of penumbral tissue loss (\%) & $1.3(0-19.9)$ & $38.4(4.9-95.3)$ & $Z=-4.23$ & $<.001^{\mathrm{c}}$ \\
\hline Infarct volume at $24 \mathrm{hr}(\mathrm{mL})$ & $4.2(1.0-9.9)$ & $38.8(11.5-107.0)$ & $Z=-5.54$ & $<.001^{\mathrm{c}}$ \\
\hline
\end{tabular}

Note:-SBP indicates systolic blood pressure; DBP, diastolic blood pressure; ONT, onset-to-needle time.

a Table cells express results in mean \pm SD for normally distributed continuous variables, No. (\%) for dichotomous variables, and median (interquartile range) for ordinal variables and non-normally distributed continuous variables, respectively.

${ }^{\mathrm{b}}$ Proportion of Penumbral Tissue Loss $=100 \times$ (Posttreatment Infarct Volume - Pretreatment Infarct Volume)/(Pretreatment Hypoperfusion Volume - Pretreatment Infarct Volume).

${ }^{c} p<.05$.

TSV, and ICV. Cases with differences of $>3 \%$ for SDR were reassessed by a neuroimaging physician (W.D.). The average value of the 2 SDRs was used.

\section{Radiologic and Clinical Assessment}

Diffusion lesion volume was calculated by applying a signal-intensity threshold of the apparent diffusion coefficient of $<600$ to pretreatment and 24-hour follow-up DWI. ${ }^{11}$ For patients with CT follow-up, the area of hypoattenuation was manually outlined. Volumetric analysis was performed by using the commercial software MIStar (Apollo Medical Imaging Technology, Melbourne, Australia). Hypoperfusion was defined as time-tomaximum of $>6$ seconds. Reperfusion was assessed in patients with a baseline hypoperfusion volume of $>10 \mathrm{~mL}$. Successful reperfusion was defined as $\geq 70 \%$ reduction in hypoperfusion volume between pretreatment and 24-hour perfusion imaging. ${ }^{12}$ The proportion of penumbral tissue loss was defined as described previously: $100 \times$ (Posttreatment Infarct Volume - Pretreatment Infarct Volume)/(Pretreatment Hypoperfusion Volume Pretreatment Infarct Volume) ${ }^{13}$

Large-artery occlusion was defined as occlusion of the internal carotid, proximal middle cerebral (M1), or proximal posterior cerebral (P1) artery. The modified Rankin Scale score was assessed at 3 months and dichotomized into good (0-2) and poor (3-6) outcomes.

\section{Statistical Analysis}

All metric and normally distributed variables were reported as mean \pm SD; non-normally distributed variables, as median (25th75 th percentile). Categoric variables were presented as frequency (percentage). The Fisher exact test was used to compare dichoto- mous variables between groups; the $t$ test, for normally distributed continuous variables; the Mann-Whitney $U$ test, for nonnormally distributed continuous variables; and the Pearson $\chi^{2}$ test, for categoric data. The Benjamini-Hochberg correction was used to control the false discovery rate of multiple comparisons. The interrater reliability was assessed by use of the intraclass correlation coefficient. The Spearman correlation coefficient was used to analyze the association of SDR in each vein with outcomes. The receiver operating characteristic curve analysis was performed, and the optimal threshold for deep vein asymmetry was determined by using the Youden index. The Mann-Whitney $U$ test was used to analyze the relationship between the asymmetry of deep veins and the proportion of penumbral loss. Univariate and multivariate logistic regression analyses were conducted to investigate whether asymmetry of the deep veins was independently associated with clinical outcome. Results are reported as odds ratios with $95 \%$ confidence intervals. A $P$ value $<.05$ was considered statistically significant. All statistical analyses were conducted by using SPSS, Version 19.0 (IBM, Armonk, New York).

\section{RESULTS}

\section{Patient Characteristics}

A total of 109 patients was included in the final analysis. The mean age was $68 \pm 13$ years with $40(36.7 \%)$ women. The mean pretreatment NIHSS score was 7 (interquartile range, 4-14), the median time from onset to MR imaging was 193 minutes (interquartile range, 144-245 minutes), and the median time from onset to treatment was 230 minutes (interquartile range, 180-283 minutes). At 3-month follow-up, 41 (37.6\%) had poor outcomes. 


\begin{tabular}{|c|c|c|c|c|}
\hline & IPTSV $(n=35)$ & Non-IPTSV $(n=74)$ & Test Value & $P$ Value \\
\hline Age (yr) & $68.9 \pm 12.8$ & $68.0 \pm 13.0$ & $t=-0.34$ & .74 \\
\hline Female (No.) (\%) & $10(28.6)$ & $30(40.5)$ & $\chi^{2}=1.47$ & .29 \\
\hline \multicolumn{5}{|l|}{ Risk factors } \\
\hline Hypertension (No.) (\%) & $28(80.0)$ & $47(63.5)$ & $\chi^{2}=3.01$ & .12 \\
\hline Diabetes mellitus (No.) (\%) & $9(25.7)$ & $16(21.6)$ & $\chi^{2}=0.23$ & .63 \\
\hline Atrial fibrillation (No.) (\%) & $13(37.1)$ & $29(39.2)$ & $\chi^{2}=0.04$ & 1.00 \\
\hline Smoking (No.) (\%) & $18(51.4)$ & $25(33.8)$ & $\chi^{2}=3.10$ & .10 \\
\hline Previous stroke/TIA (No.) (\%) & $6(17.1)$ & $11(14.9)$ & $\chi^{2}=0.10$ & .78 \\
\hline Large-artery occlusion (No.) (\%) & $26(74.3)$ & $34(45.9)$ & $\chi^{2}=7.02$ & $.007^{\mathrm{e}}$ \\
\hline Baseline SBP (mm Hg) & $153.7 \pm 20.0$ & $153.7 \pm 23.4$ & $t=0.01$ & .99 \\
\hline Baseline DBP (mm Hg) & $83.1 \pm 10.7$ & $86.8 \pm 18.3$ & $t=1.32$ & .19 \\
\hline ONT (min) & $266.9 \pm 68.6$ & $229.0 \pm 73.8$ & $t=-2.56$ & $.01^{\mathrm{e}}$ \\
\hline Baseline glucose level (mmol/L) & $7.7 \pm 2.1$ & $7.9 \pm 2.9$ & $t=0.42$ & .67 \\
\hline Baseline NIHSS score & $13.0(7.0-18.0)$ & $7.0(3.8-11.5)$ & $Z=-2.87$ & $.004^{\mathrm{e}}$ \\
\hline Baseline diffusion lesion volume (mL) & $5.9(0.9-65.9)$ & $2.0(0-15.1)$ & $Z=-2.74$ & $.006^{\mathrm{e}}$ \\
\hline Baseline hypoperfusion volume (mL) & $111.2(44.0-173.3)$ & $29.3(5.0-71.4)$ & $Z=-4.04$ & $<.001^{\mathrm{e}}$ \\
\hline $24-\mathrm{Hr}$ CT scan (No.) (\%) & $1(2.9)$ & $3(4.3)$ & $\chi^{2}=0.096$ & 1.00 \\
\hline Proportion of penumbral tissue loss $(\%)^{c}$ & $32.6(0.6-100.0)$ & $4.5(0-27.4)$ & $Z=-2.89$ & $.004^{\mathrm{e}}$ \\
\hline Infarct volume at 24 hours (mL) & $40.8(7.5-113.6)^{\prime}$ & $5.0(1.0-19.6)$ & $Z=-4.27$ & $<.001^{\mathrm{e}}$ \\
\hline Poor outcome (No.) (\%) ${ }^{d}$ & $22(62.9)$ & $19(25.7)$ & $\chi^{2}=14.00$ & $<.001^{\mathrm{e}}$ \\
\hline
\end{tabular}

Note:-SBP indicates systolic blood pressure; DBP, diastolic blood pressure; ONT, onset-to-needle time.

a Table cells express results in mean \pm SD for normally distributed continuous variables, No. (\%) for dichotomous variables, and median (interquartile range) for ordinal variables and non-normally distributed continuous variables, respectively.

b Twenty-four-hour CT scan: the proportion of patients imaged with CT rather than MRI for 24-hour follow-up imaging.

${ }^{c}$ Proportion of Penumbral Tissue Loss $=100 \times$ (Posttreatment Infarct Volume - Pretreatment Infarct Volume)/(Pretreatment Hypoperfusion Volume - Pretreatment Infarct Volume).

d Poor outcome $=3$-month modified Rankin Scale score $\geq 2$.

${ }^{e} p<.05$.

Table 3: Multivariate regression analysis for poor outcome

\begin{tabular}{llcc}
\hline & OR & $\mathbf{9 5 \%} \mathbf{C l}$ & $\boldsymbol{P}$ Value \\
\hline Age & 1.04 & $1.00-1.09$ & .04 \\
Baseline NIHSS score & 1.11 & $1.03-1.20$ & .009 \\
Atrial fibrillation & 1.66 & $0.60-4.60$ & .33 \\
Diabetes mellitus & 1.99 & $0.64-6.18$ & .24 \\
Large-artery occlusion & 1.99 & $0.56-7.03$ & .29 \\
Baseline hypoperfusion volume & 1.00 & $0.99-1.01$ & .68 \\
IPTSV & 3.66 & $1.25-10.68$ & .02 \\
\hline
\end{tabular}

\section{Deep Cerebral Veins and Outcome}

Interrater agreement was excellent for the SDR of the SV (intraclass correlation coefficient $=0.82$ ), TSV (intraclass correlation coefficient $=0.88$ ), and ICV (intraclass correlation coefficient $=$ $0.90)$. The SDR of the TSV was correlated with poor outcome ( $\rho=$ $0.25, P=.008)$, but there was no correlation for the SV $(\rho=0.04$, $P=.73)$ or ICV $(\rho=0.07, P=.47)$. Patients with poor outcome had a higher SDR of the TSV (median, $4.5 \%$, versus $0.7 \% ; Z=$ $-2.61, P=.009$ ) (Table 1). Receiver operating characteristic analysis revealed that the SDR of the TSV was associated with poor outcome (area under the curve $=0.65 ; 95 \% \mathrm{CI}, 0.54-0.76 ; P=$ .009 ), with the optimal threshold of $4.8 \%$ for identifying poor outcome (sensitivity of $53.7 \%$ and specificity of $80.9 \%$, Youden index $=0.35)$.

\section{Ipsilateral Prominent Thalamostriate Vein and Outcome}

On the basis of the cutoff of $4.8 \%$, we then defined ipsilateral prominent TSV (IPTSV) as an SDR of the TSV of $\geq 5 \%$ and dichotomized patients into IPTSV and non-IPTSV groups. As seen in Table 2, univariate analysis showed that patients with IPTSV had a higher rate of large-artery occlusion (74.3\% versus $45.9 \%$, $P=.007$ ), higher baseline NIHSS scores (median, 13 versus 7; $P=$
$.004)$, and longer onset-to-needle time $(266.9 \pm 68.6$ minutes versus $229.0 \pm 73.8$ minutes, $P=.01)$, compared with those with non-IPTSV. Baseline hypoperfusion volume was independently associated with the presence of IPTSV (OR $=1.01 ; 95 \%$ CI, 1.00 1.02; $P=.02$ ) after adjusting for large-artery occlusion, NIHSS score, and onset-to-needle time.

Patients with IPTSV had significantly higher rates of poor outcome than those with non-IPTSV (62.9\% versus $25.7 \%$ ), which remained significant in multivariate regression, which included age, NIHSS score, atrial fibrillation, diabetes, large-artery occlusion status, and baseline hypoperfusion volume ( $\mathrm{OR}=3.66 ; 95 \%$ CI, 1.25-10.68; $P=.02$ ) (Table 3 and Fig 2). The presence of IPTSV was associated with a high proportion of penumbral tissue loss (32.6\% versus $4.5 \%, P=.004)$.

\section{Relationship between Ipsilateral Prominent Thalamostriate Vein and Reperfusion}

Reperfusion status was evaluated in 77 patients. The presence of the IPTSV was associated with a lower successful reperfusion rate (37.5\% versus $60.0 \%$; OR $=0.35 ; 95 \%$ CI, $0.13-0.92 ; P=.03$ ) after adjusting for baseline diastolic blood pressure and onset-toneedle time.

In the subgroup of patients without reperfusion, patients with IPTSV had a significantly higher rate of poor outcome $(80.0 \%$ versus $44.4 \%, P=.04$ ) compared with those without IPTSV. IPTSV was not associated with poor functional outcome in patients with successful reperfusion $(41.7 \%$ versus $25.9 \%, P=.46)$ (Fig 3).

\section{DISCUSSION}

Our study quantitatively described the relationship between deep cerebral veins and neurologic outcomes after IVT based on SWI 

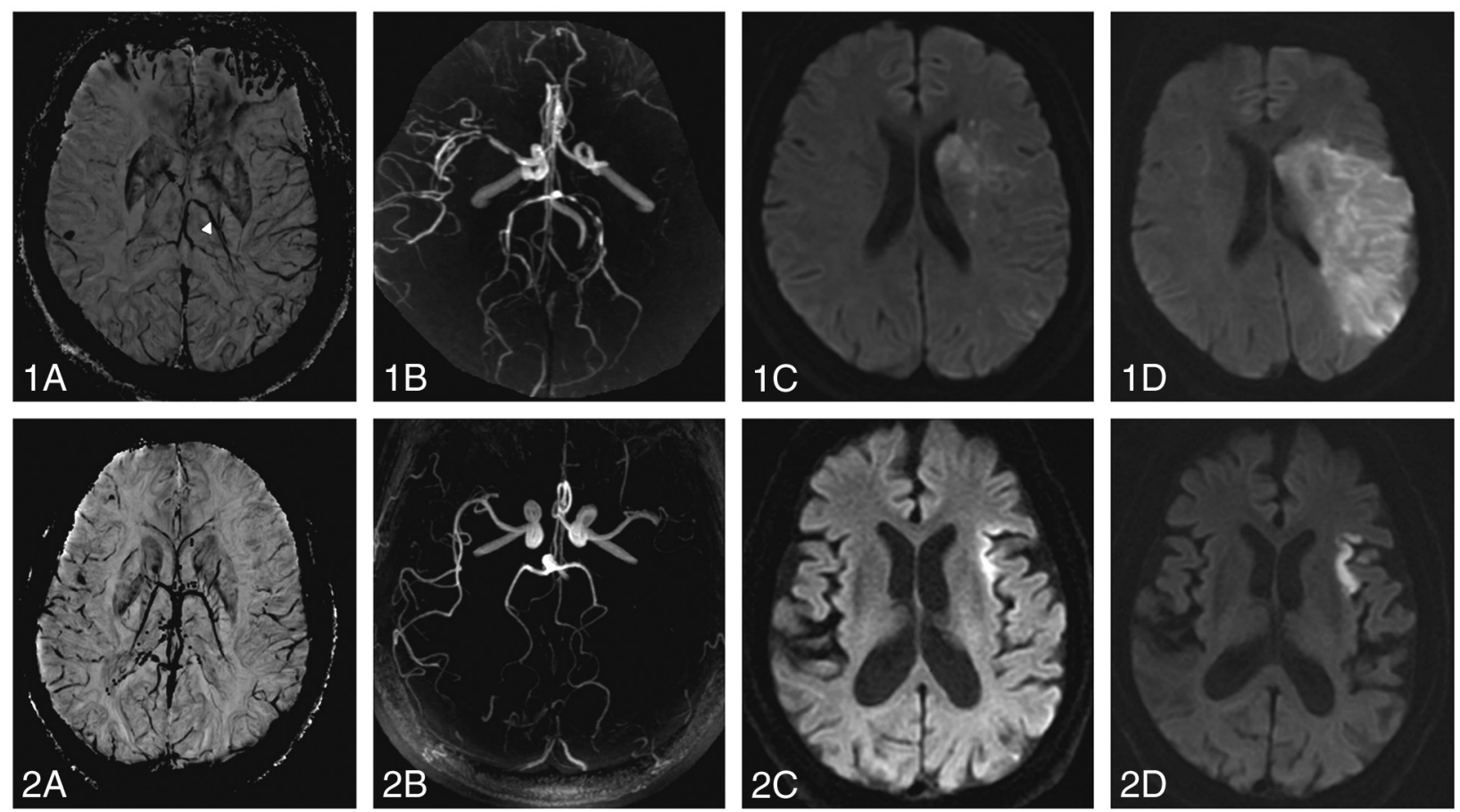

FIG 2. Outcome in patients with and without an ipsilaterally prominent thalamostriate vein. Two illustrative cases: a 55 -year old female patient with left-sided thromboembolic occlusion of the M1 segment (1B) and IPTSV (1A, arrow) who developed major infarct growth between pretreatment (1C) and 24-hour postthrombolysis imaging (ID). The 3-month modified Rankin Scale score was 5. A 54-year old male patient with left-sided thromboembolic occlusion of the M1-segment (2B) without IPTSV (2A) who had similar pretreatment diffusion lesion volume (2C) but less infarct growth at 24 hours $(2 D)$. The 3-month mRS was 1 .

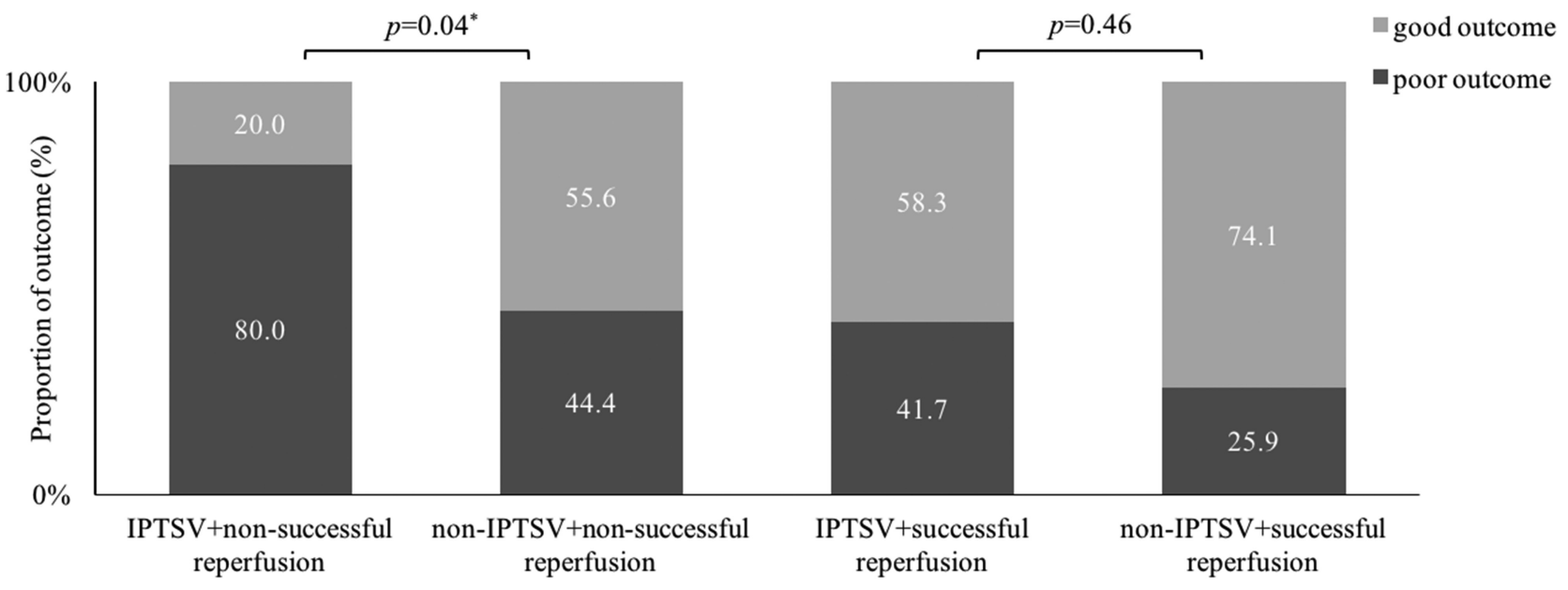

FIG 3. Relationship between categories of outcome with reperfusion and the presence of the ipsilaterally prominent thalamostriate vein. Note successful reperfusion is $\geq 70 \%$ reduction in hypoperfusion volume between pretreatment and 24 hours. Poor outcome is a 3-month modified Rankin Scale score $\geq 2$. Asterisk indicates $P<.05$.

and revealed that the presence of IPTSV (defined as an SDR of the TSV of $\geq 5 \%$ ) was strongly associated with poor outcome after IVT. Without reperfusion, $>80 \%$ of patients with IPTSV had poor outcomes, while this rate decreased to $42 \%$ if reperfused, which reinforced the importance of reperfusion treatment in patients with IPTSV.

In the current study, we found an association only between poor outcome and SDR of the TSV and not the SV or ICV. This may reflect the functional impact of the brain regions that drain to each vein. The TSV mainly drains the basal ganglia and thalamus, which play important roles in motor control and motor learn- ing, ${ }^{14-16}$ and these functions have a strong influence on the mRS score. ${ }^{17}$ Lesions within the territory draining to the SV, such as the septum pellucidum, rostral corpus callosum, and white matter of the frontal lobe, may have less impact on the mRS score. The ICV receives tributaries from subcortical, periventricular structures and the choroid plexus within the lateral ventricle, and this extensive venous drainage territory may dilute the relationship between critical functions and changes in venous signal.

The presence of IPTSV was associated with a larger hypoperfusion volume at baseline. This finding is consistent with a previous study that demonstrated that prominent deep cerebral veins 
on gradient-echo $\mathrm{T} 2{ }^{\star}$-weighted imaging correlated with a large perfusion lesion. ${ }^{18}$ When the oxygen extraction fraction is elevated in hypoperfused tissue, the increased level of deoxyhemoglobin is reflected in abnormal hypointensity of the veins draining that region of the brain. Furthermore, we found that patients with IPTSV were likely to have a high proportion of penumbral tissue loss at 24-hour follow-up. The ischemic penumbra represents the potentially salvageable hypoperfused region, ${ }^{19-21}$ which retains structural integrity but has lost function after AIS. Without timely reperfusion, the penumbra becomes irreversibly damaged. Recent studies interpreted the presence of extensive prominent cortical vessels and asymmetry of deep medullary veins after AIS as evidence of poor leptomeningeal collateralization, because good collateralization should lead to less deoxygenated blood in the veins. ${ }^{22-24}$ Collateral status is one of the most important factors determining penumbral tissue loss, and good collaterals have been associated with reduced penumbral loss. ${ }^{13,25}$ Therefore, we speculated that the presence of IPTSV might indicate poor collaterals after acute ischemia, which would result in increased penumbral tissue loss. However, it is challenging to directly investigate the relationship between IPTSV and leptomeningeal collaterals in our data because we included patients with both largeartery occlusion and more distal occlusions. Further studies are required to explore this.

Our data also showed that patients with IPTSV were less likely to achieve reperfusion. Experimental studies have demonstrated venous endothelial injury and subsequent clot formation in cerebral veins due to oxidative stress and inflammation in the upstream ischemic area. ${ }^{26}$ Occlusion of microthrombi in venules was found within 30 minutes after acute reduction of CBF in mice, leading to decreased venous flow velocity. ${ }^{27}$ It is possible that the decreased venous outflow could result in an increase in intracranial pressure as blood accumulates in the capillary system. Subsequently, increased intracranial pressure may lead to decreased arterial perfusion, particularly in collateral pathways, and may contribute to the evolution of penumbral tissue into infarct. ${ }^{28}$

Successful reperfusion appeared beneficial to patients with IPTSV, reducing the rate of poor outcome from $80 \%$ to $42 \%$. This finding indicates the presence of salvageable penumbra in patients with IPTSV. Intra-arterial thrombectomy has been established as a more effective reperfusion therapy in patients with documented occlusion of the distal internal carotid or proximal middle cerebral artery. ${ }^{29,30}$ Our results may not directly affect clinical decision-making; however, further studies are required to evaluate whether patients with IPTSV are particularly likely to benefit from thrombectomy in addition to thrombolysis.

Our study has several limitations. First, it is a retrospective analysis and has a potential risk of selection bias. However, consecutive patients were included in a prospective stroke registry from our stroke center with highly homogeneous and standardized medical care. Second, both CT and MR imaging were used for follow-up imaging, which may increase the heterogeneity of volume measurement. However, the proportion of follow-up CT was $<5 \%$ and was similar in patients with and without IPTSV. Third, we did not evaluate the follow-up status of veins due to the variable follow-up imaging, and this will be the subject of future study. Fourth, chronic arterial stenosis in cervicocranial arteries can result in preexisting abnormal signal in deep veins before an acute ischemic event. This may influence the assessment of asymmetry of the deep venous system after AIS. Our results should be validated in patients with stenosis of the internal carotid artery and intracranial arteries in further investigations. Finally, our conclusions were based on patients receiving IVT. Future studies are needed to validate the use of IPTSV in the context of endovascular thrombectomy.

\section{CONCLUSIONS}

Patients with IPTSV had a lower incidence of reperfusion and a higher rate of poor outcome after IVT. The risk of poor outcome was, however, markedly reduced in those patients in whom successful reperfusion was achieved. Our results emphasize the importance of reperfusion, especially for the patients with IPTSV. However, further studies are warranted to validate whether patients with IPTSV can benefit from more successful reperfusion strategies.

Disclosures: Min Lou—RELATED: Grant: Science Technology Department of Zhejiang Province (2013C13G2010032) and the National Natural Science Foundation of China (81471170 \& 81622017).* *Money paid to the institution.

\section{REFERENCES}

1. Chen CY, Chen CI, Tsai FY, et al. Prominent vessel sign on susceptibility-weighted imaging in acute stroke: prediction of infarct growth and clinical outcome. PLoS One 2015;10:e131118 CrossRef Medline

2. Baik SK, Choi W, Oh SJ, et al. Change in cortical vessel signs on susceptibility-weighted images after full recanalization in hyperacute ischemic stroke. Cerebrovasc Dis 2012;34:206-12 CrossRef Medline

3. Sun W, Liu W, Zhang Z, et al. Asymmetrical cortical vessel sign on susceptibility-weighted imaging: a novel imaging marker for early neurological deterioration and unfavorable prognosis. Eur J Neurol 2014;21:1411-18 CrossRef Medline

4. Zaitsu Y, Kudo K, Terae S, et al. Mapping of cerebral oxygen extraction fraction changes with susceptibility-weighted phase imaging. Radiology 2011;261:930-36 CrossRef Medline

5. Santhosh K, Kesavadas C, Thomas B, et al. Susceptibility weighted imaging: a new tool in magnetic resonance imaging of stroke. Clin Radiol 2009;64:74-83 CrossRef Medline

6. Robertson CA, McCabe C, Gallagher L, et al. Stroke penumbra defined by an MRI-based oxygen challenge technique, 1: validation using [14C]2-deoxyglucose autoradiography. J Cereb Blood Flow Metab 2011;31:1778-87 CrossRef Medline

7. De Crespigny AJ, Wendland MF, Derugin N, et al. Real-time observation of transient focal ischemia and hyperemia in cat brain. Magn Reson Med 1992;27:391-97 CrossRef Medline

8. Fujioka M, Okuchi K, Iwamura A, et al. A mismatch between the abnormalities in diffusion- and susceptibility-weighted magnetic resonance imaging may represent an acute ischemic penumbra with misery perfusion. J Stroke Cerebrovasc Dis 2013;22:1428-31 CrossRef Medline

9. Huang $\mathrm{P}$, Chen $\mathrm{CH}$, Lin WC, et al. Clinical applications of susceptibility weighted imaging in patients with major stroke. J Neurol 2012;259:1426-32 CrossRef Medline

10. Doshi H, Wiseman N, Liu J, et al. Cerebral hemodynamic changes of mild traumatic brain injury at the acute stage. PLoS One 2015;10: e0118061 CrossRef Medline

11. Purushotham A, Campbell BC, Straka M, et al. Apparent diffusion coefficient threshold for delineation of ischemic core. Int J Stroke 2015;10:348-53 CrossRef Medline 
12. Zhang S, Tang H, Yu YN, et al. Optimal magnetic resonance perfusion thresholds identifying ischemic penumbra and infarct core: a Chinese population-based study. CNS Neurosci Ther 2015;21: 289-95 CrossRef Medline

13. Jung S, Gilgen M, Slotboom J, et al. Factors that determine penumbral tissue loss in acute ischaemic stroke. Brain 2013;136(pt 12): 3554-60 CrossRef Medline

14. DeLong MR, Alexander GE, Mitchell SJ, et al. The contribution of basal ganglia to limb control. Prog Brain Res 1986;64:161-74 CrossRef Medline

15. Mink JW, Thach WT. Basal ganglia motor control. III. Pallidal ablation: normal reaction time, muscle cocontraction, and slow movement. J Neurophysiol 1991;65:330-51 Medline

16. Wichmann T, DeLong MR. Functional and pathophysiological models of the basal ganglia. Curr Opin Neurobiol 1996;6:751-58 CrossRef Medline

17. Cheng B, Forkert ND, Zavaglia M, et al. Influence of stroke infarct location on functional outcome measured by the modified Rankin scale. Stroke 2014;45:1695-702 CrossRef Medline

18. Hermier M, Nighoghossian N, Derex L, et al. Hypointense transcerebral veins at $\mathrm{T} 2{ }^{*}$-weighted $\mathrm{MRI}$ : a marker of hemorrhagic transformation risk in patients treated with intravenous tissue plasminogen activator. J Cereb Blood Flow Metab 2003;23:1362-70 CrossRef Medline

19. Meoded A, Poretti A, Benson JE, et al. Evaluation of the ischemic penumbra focusing on the venous drainage: the role of susceptibility weighted imaging (SWI) in pediatric ischemic cerebral stroke. J Neuroradiol 2014;41:108-16 CrossRef Medline

20. Mucke J, Möhlenbruch M, Kickingereder P, et al. Asymmetry of deep medullary veins on susceptibility weighted MRI in patients with acute MCA stroke is associated with poor outcome. PLoS One 2015; 10: e0120801 CrossRef Medline
21. Phan TG, Wright PM, Markus R, et al. Salvaging the ischaemic penumbra: more than just reperfusion? Clin Exp Pharmacol Physiol 2002;29:1-10 CrossRef Medline

22. Oh MY, Ko SB, Kim CK, et al. Abstract 13: Asymmetry of deep medullary veins in susceptibility weighted imaging is associated with poor collaterals in patients with acute ischemic stroke. Stroke 2013; 44(suppl 1):A13

23. Verma RK, Hsieh K, Gratz PP, et al. Leptomeningeal collateralization in acute ischemic stroke: impact on prominent cortical veins in susceptibility-weighted imaging. Eur J Radiol 2014;83: 1448-54 CrossRef Medline

24. Parthasarathy R, Sohn SI, Jeerakathil T, et al. A combined arterial and venous grading scale to predict outcome in anterior circulation ischemic stroke. J Neuroimaging 2015;25:969-77 CrossRef Medline

25. Bang OY, Saver JL, Buck BH, et al; UCLA Collateral Investigators. Impact of collateral flow on tissue fate in acute ischaemic stroke. J Neurol Neurosurg Psychiatry 2008;79:625-29 Medline

26. Zhou Y, Wang Y, Wang J, et al. Inflammation in intracerebral hemorrhage: from mechanisms to clinical translation. Prog Neurobiol 2014;115:25-44 CrossRef Medline

27. Schwarzmaier SM, Kim SW, Trabold R, et al. Temporal profile of thrombogenesis in the cerebral microcirculation after traumatic brain injury in mice. J Neurotrauma 2010;27:121-30 CrossRef Medline

28. Li Q, Khatibi N, Zhang JH. Vascular neural network: the importance of vein drainage in stroke. Transl Stroke Res 2014;5:163-66 CrossRef Medline

29. Berkhemer OA, Fransen PS, Beumer D, et al. A randomized trial of intraarterial treatment for acute ischemic stroke. N Engl J Med 2015; 372:11-20 CrossRef Medline

30. Goyal M, Demchuk AM, Menon BK, et al; ESCAPE Trial Investigators. Randomized assessment of rapid endovascular treatment of ischemic stroke. $N$ Engl J Med 2015;372:1019-30 CrossRef Medline 